\title{
(1)APO
}

\section{Remodelação estética de dentes conoides: tratamento multidisciplinar}

\author{
Esthetic remodeling of conoid teeth - multidisciplinary treatment
}

\author{
Maria Cecília Veronezi ${ }^{1}$ \\ Letícia Ferreira de Freitas Brianezzi ${ }^{2}$ \\ Karin Modena ${ }^{1}$ \\ Maria Silvia de Lima $^{3}$ \\ Silvana Elizabeth Bernardi ${ }^{4}$
}

\section{RESUMO}

A procura pela estética para se obter um sorriso saudável e atrativo tem aumentado. O objetivo desse artigo é relatar um caso de dois incisivos laterais com morfologia conoide e diastemas com distribuição desproporcional, que teve remodelação estética por meio de movimentação ortodôntica, aumento de coroa e resina composta. Uma paciente jovem procurou tratamento porque estava insatisfeita com seu sorriso, devido à presença de dentes conoides e diastemas. Além disso, a harmonia do sorriso era afetada pela presença de um desnível do zênite gengival dos incisivos centrais. Para planejamento do caso, foi realizado um modelo diagnóstico e observada a necessidade de movimentação ortodôntica, para redistribuição dos espaços presentes nos diastemas, e cirurgia periodontal, para elevar a margem gengival dos incisivos centrais. Após o período de preparo, foi feito clareamento caseiro e posteriormente o tratamento restaurador com resina composta. Após o controle de 7 meses, pode-se observar ausência de inflamação gengival, além de um sorriso harmônico e estético.

Palavras-chaves: Incisivo. Diastemas. Estética.

\begin{abstract}
The seek for aesthetics to obtain a healthy and attractive smile has increased. The aim of this article is to report a case of two lateral incisors with conoid morphology and diastemas with disproportionate distribution, which had aesthetic remodeling through orthodontic movement, surgical crown lenghtening and composite resin restoration. A young patient sought treatment because she was unsatisfied with her smile, due to the presence of conoid teeth and diastema. In addition, the smile harmony was affected by the presence of a gingival zenith misalignment of the central incisors. For planning of the case, a diagnostic model was performed and a need for orthodontic movement was observed for the redistribution of diastema spaces and periodontal surgery to raise the gingival margin of the central incisors. After the preparation period, an at-home bleaching was performed followed by the restorative treatment with composite resin. After the control of 7 months, one can observe absence of gingival inflammation, besides a harmonic and aesthetic smile.
\end{abstract}

Keywords: Incisor. Diastema. Aesthetics

\footnotetext{
${ }^{1}$ Professora Doutora, Curso de Odontologia, Universidade Sagrado Coração de Jesus

${ }^{2}$ Doutoranda, Faculdade de Odontologia de Bauru, Universidade de São Paulo

${ }^{3}$ Professora Mestre, Instituto Cecília Veronezi

${ }^{4}$ Mestre, Faculdade de Odontologia de Ribeirão Preto, Universidade de São Paulo
} 


\section{INTRODUÇão}

Um segmento muito importante e dominante na composição estética dento-facial de um indivíduo é o sorriso. Discrepâncias no tamanho, forma, cor, posição dos dentes, presença de diastemas e dentes conoides tem um impacto, não apenas na harmonia da estética dental, mas também do conjunto estético dento-facial e da autoestima dos pacientes ${ }^{1,2}$.

Uma situação que tem se tornado cada vez mais comum e, afeta negativamente na beleza do sorriso, é a presença de incisivos laterais conoides e diastemas, que em adultos confere um sorriso infantil e está fora dos padrões de beleza atuais. Essa alteração de desenvolvimento dental está relacionada com o tamanho e formato do dente e é classificada como microdontia isolada ${ }^{3,4}$. Normalmente acometem com mais frequência o gênero feminino, incidindo em cerca de 1,03\% da população e ocorrendo em ambos os lados da arcada dentária, sendo o incisivo lateral superior o elemento mais afetado por essa alteração ${ }^{5-7}$.

Em alguns casos, para se obter um resultado satisfatório, há necessidade de um planejamento estético-funcional envolvendo a integração de diversas especialidades da odontologia ${ }^{2}$ tais como: periodontia, ortodontia e dentística. Devido à evolução de novas técnicas e materiais restauradores, as opções de tratamento restaurador de incisivos laterais conoides foram ampliadas. Dente elas, destacamos a remodelação estética de maneira rápida, econômica e conservativa, através das resinas compostas ${ }^{8}$.

Recentemente, as facetas de cerâmicas minimamente invasivas ou "lentes de contato" entraram no mercado odontológico e se tornaram uma alternativa atraente para resolver o problema estético de dentes conoides. Contudo, devido ao alto custo do material utilizado, grande parte da população não tem acesso a esse tipo de tratamento, principalmente nos dias de hoje. Desse modo, as restaurações diretas com resina composta são uma excelente alternativa para solucionar esses casos. No entanto, requerem maior habilidade e conhecimento do cirurgião dentista para reproduzir o aspecto natural dos dentes ${ }^{8,9}$.
Sendo assim, o objetivo do presente artigo é relatar o tratamento de uma paciente com incisivos laterais superiores conoides e diastemas, onde uma abordagem multidisciplinar envolvendo as áreas de ortodontia, periodontia e dentística foram empregadas para remodelação estética.

\section{Relato de Caso}

Paciente jovem, do gênero feminino apresentava queixa do formato e presença de diastemas nos incisivos laterais (Figuras 1 e 2). Durante a avalição clínica, detectou-se desproporção no comprimento/ largura dos incisivos centrais superiores, a qual era provocada pela arquitetura periodontal com o zênite gengival destes elementos mais para incisal, em relação aos dos incisivos laterais e caninos (Figura 1).

Para planejamento do caso foi realizado um modelo diagnóstico, onde se observou a necessidade de redistribuir melhor os espaços dos diastemas presentes nos incisivos laterais superiores, por meio tratamento ortodôntico. Após a finalização do tratamento, o ortodontista restaurou provisoriamente os incisivos laterais conoides para a manutenção do espaço até a realização da remodelação estética (Figura 3).

Antes da cirurgia periodontal, foi realizado um enceramento para ser utilizado como guia cirúrgico durante o aumento de coroa. Após a cirurgia, podemos observar o restabelecimento de um dos padrões gengivais considerado estético, que é a margem gengival dos incisivos centrais, laterais e caninos na mesma altura.

Após 90 dias da cirurgia periodontal, foi realizado o clareamento caseiro (Figura 3) com gel peróxido de carbamida 16\% (Whithness Blue, FGM, Joinville, SC, BR) em 2 semanas, passando da cor A3,5 para A2. Após 15 dias do procedimento clareador, foi realizado o procedimento restaurador estético. Como visto no enceramento diagnóstico (Figura 4), além da reanatomização dos incisivos laterais foi adicionado resina composta nas incisais e distais dos incisivos centrais, e mesiais dos caninos.

Para a remodelação foi realizado isolamento absoluto do campo operatório, utilizando amarrias com 
fio dental com a finalidade de promover melhor afastamento gengival. Durante o procedimento restaurador foi utilizado um guia de silicona (Zetalabor, Zhermack, Badia Polesine, IT) que foi obtido através do enceramento diagnóstico (Figura 5). O preparo mecânico dos elementos dentais consistiu apenas na asperização com pontas diamantadas em velocidade baixa a média.

No processo de hibridização, foi aplicado sobre o esmalte ácido fosfórico a 37\% (Ultradent, Indaiatuba, SP, BR) e sistema adesivo (Single Bond Universal, 3M ESPE, St. Paul, MN, USA). As conchas palatinas foram confeccionadas com a resina composta WE (Z350 XT, 3M ESPE ${ }^{\circledR}$ ). Utilizou-se um guia de silicona para reconstrução da anatomia palatina e da borda incisal (Figura 5). Na porção de dentina foi utilizada resina A2 de dentina (Premisa, Kavo Kerr, Joinville, SC, BR), além disso, foram esculpidos os lóbulos e as depressões de desenvolvimento (Figura 6). Em seguida, foi inserido resina de esmalte A2 (Empress Direct, Ivoclar, Barueri, SP, BR) no terço médio e cervical, sem recobrir totalmente os mamelos (Figura 6). Para criar efeito natural, reproduzindo a translucidez que a paciente apresentava nos demais elementos, foi utilizada a resina IrB (Vitalescense, Ultradent ${ }^{\circledR}$ ) entre e ao redor dos mamelos (Figura 7). Para finalizar a reconstrução, no terço incisal, foi utilizada a resina Renamel (Cosmedent, Chicago, IL, USA) na cor IM conseguindo assim translucidez suficiente para a visualização de toda a caracterização deste terço. Em cada passo, a polimerização foi feita com fotopolimerizador LED (Valo, Ultradent ${ }^{\circledR}$ ) por 40 segundos.

Ao final do procedimento foi realizado ajuste oclusal e acabamento imediato com lâminas de bisturi $\mathrm{n}^{\mathrm{o}} 12$ e disco de lixa de granulação grossa (Soft Lex Pop On, 3M ESPE ${ }^{\circledR}$ ) (Figura 8). Para acabamento e polimento final, empregou-se discos abrasivos de granulação média e fina (Soft Lex Pop On, 3M ESPE ${ }^{\circledR}$ ) para reproduzir a anatomia primária e deixar os dentes simétricos, borrachas tipo enhance (Dentsply, Petrópolis, RJ, BR) escova de carbeto de silício $\left(\right.$ Cosmedent $\left.^{\circledR}\right)$, pasta diamantada (Enamelize,
Cosmedent ${ }^{\circledR}$ ) obtendo-se um resultado final altamente satisfatório. A Figura 9 mostra o aspecto final e controle de 7 meses.

\section{Discussão}

Compreende-se que um sorriso harmônico tem grande influência na vida e na autoestima das pessoas, devido a isso procedimentos estéticos têm sido cada vez mais solicitados nos consultórios odontológicos, visando um sorriso "perfeito". Para que essa alta exigência estética seja atingida com sucesso é necessário um planejamento multidisciplinar envolvendo várias especialidades da odontologia ${ }^{1}$.

$\mathrm{Na}$ era dos softwares do planejamento digital do sorriso, a velha técnica do enceramento diagnóstico não foi totalmente substituída. O enceramento é uma ferramenta de visualização inicial útil para simular o resultado final desejado do tratamento. $\mathrm{O}$ modelo permite, ao cirurgião dentista, estudar cada dente envolvido no tratamento, observando a necessidade de reposicionamento ou desproporção altura/largura. Além disso, o enceramento, possibilita confeccionar guias para o tratamento estético e a realização de mock-up que, se bem conduzido, possibilitará ao paciente a visualização da estética materializada ${ }^{9}$.

No presente caso, inicialmente foi realizado um modelo diagnóstico para observar a necessidade da arcada e de cada dente. Nessa fase, a necessidade de realizar o tratamento ortodôntico foi observada, sendo um passo importante para corrigir a posição dos dentes e a relação interarcos, gerenciando a proporção dentária e melhorar a linha do sorriso ${ }^{1,10}$. Após a obtenção dos espaços necessários para realização da remodelação estética dos dentes, o ortodontista realizou uma restauração provisória para a manutenção dos espaços conseguidos. Sabe-se que existem outros métodos para manutenção dos espaços como a utilização de placas, mas para isto, é importante o comprometimento do paciente para que os espaços não sejam perdidos, devido a isso a restauração provisória é uma boa proposta para manutenção dos espaços. 
Com a finalização do tratamento ortodôntico, obteve-se um enceramento para confeccionar guia cirúrgico para remodelação da estética rosa. Isso facilita a realização da cirurgia e garante previsibilidade do tratamento ${ }^{11}$. Sabe-se que a estética rosa tem grande influência no sorriso e deve ser avaliada em todos os casos. Na cirurgia de aumento de coroa foi realizado retalho de espessura total associado à osteotomia, visto a necessidade de remoção de tecido ósseo para estabelecimento da proporção altura/largura e zênit dos incisivos centrais e caninos que apresentavam margem gengival abaixo dos incisivos laterais. Nos padrões gengivais considerados estéticos, a margem gengival dos incisivos centrais, laterais e caninos encontram-se na mesma altura, como observado no resultado final do presente caso, ou a ainda, margem dos incisivos centrais e caninos estarem mais apical em relação aos incisivos laterais ${ }^{12}$. Apesar do último padrão ser considerado o padrão ouro periodontal, a paciente considerava estético a margem dos dentes envolvidos na mesma altura. Além disso, para estabelecimento desse padrão os incisivos centrais e caninos ficariam muito grandes, tendo a necessidade de desgaste de estrutura dentária sadia para obtenção de um sorriso harmônico.

Posteriormente ao processo de reparo gengival, foram realizados: o clareamento com gel de peróxido de carbamida e a confecção da remodelação estética com resina composta. Compreende-se que o clareamento dental pode ser realizado em consultório ou de forma caseira, sempre com orientação do profissional. $\mathrm{O}$ clareamento caseiro apresenta a vantagem de ser autoaplicável, menor tempo clínico para o paciente e cirurgião dentista e apresenta menor custo, em relação ao clareamento de consultório, em contrapartida, no consultório, existe maior controle sob os tecidos moles e ingestão do ge $1^{13,14}$.

Sabe-se que as resinas compostas atuais apresentam ótimas propriedades ópticas e mecânicas, conferindo uma estética natural e longevidade das restaurações ${ }^{15,16,17,19}$. Além disso, apresentam a vantagem da técnica reversível, possibilidade de reparos, custo reduzido, visto que não há necessidade de trabalho laboratorial em relação às cerâmicas minimamente invasivas ou "lentes de contato" 13,14 . No entanto, a retenção do brilho é menor, mas este problema pode ser solucionado com um re-polimento periodicamente.

Nos casos em que o paciente apresenta baixas condições econômicas ou precisa de um procedimento mais rápido a resina composta é a melhor opção de tratamento. Contudo, requer um grande conhecimento sobre as cores ${ }^{15,16,18}$, principalmente nos dias de hoje, visto que existe uma grande gama de translucidez, opacidade, opalescência das resinas e o método utilizado pelas empresas para classificá-las são diferentes, podendo obter um resultado final insatisfatório. Além disso, exige habilidade manual do cirurgião dentista em manipulá-la para obter uma restauração imperceptível.

\section{Conclusão}

Conclui-se, que a remodelação estética de dentes laterais conoide exige, na maioria dos casos, um planejamento multidisciplinar para o sucesso do tratamento. Além disso, o emprego de sistemas restauradores adesivos diretos são uma ótima opção para tratamento desses casos, por permitirem um procedimento altamente conservador com ausência ou mínimo desgaste de estrutura dentária, com baixo custo, devolvendo ao paciente a estética e a harmonia do sorriso.

\section{FIGURAS}

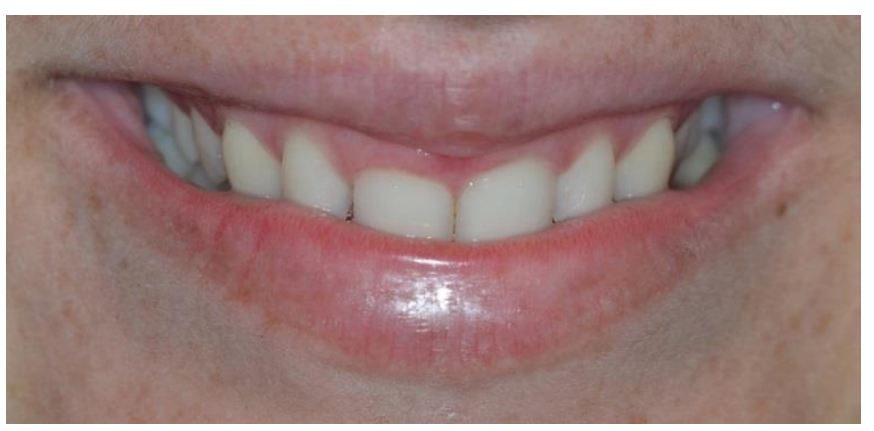

Figura 1- Sorriso inicial. Paciente apresenta sorriso alto. 


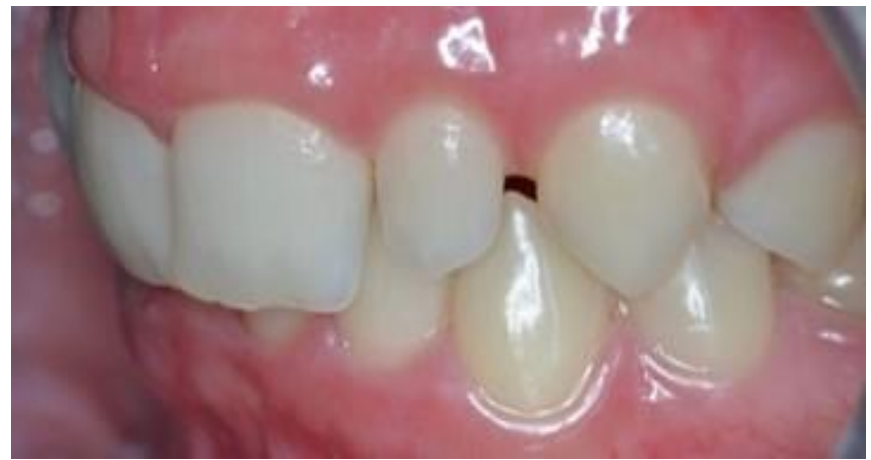

Figura 2 - Vista de um dos incisivos laterais com morfologia conoide e presença de diastemas (dente 22).

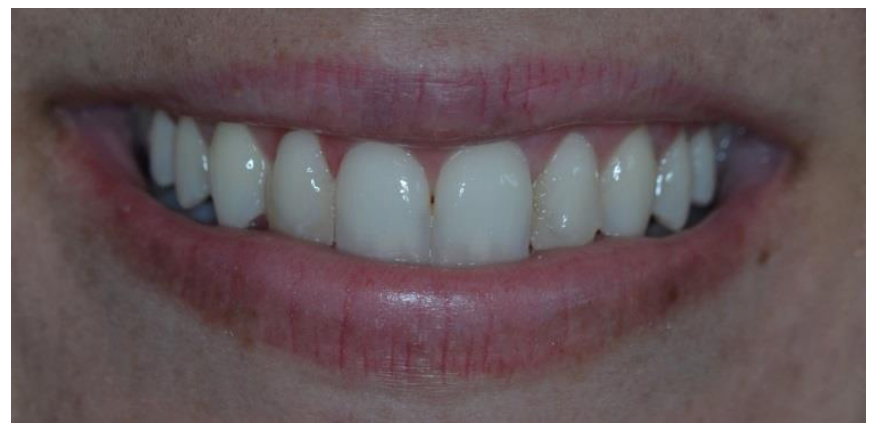

Figura 3- Sorriso após a cirurgia periodontal e clareamento caseiro.

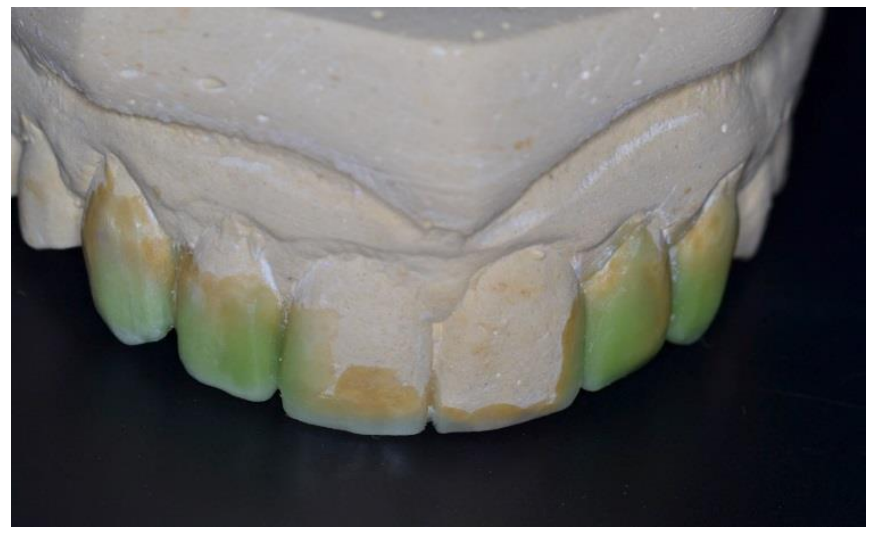

Figura 4- Enceramento diagnóstico.

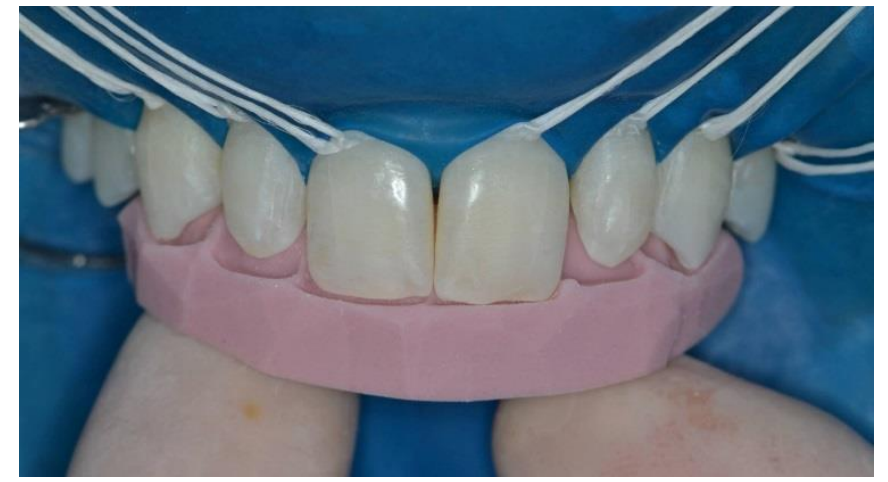

Figura 5- Após a remoção da resina provisória, foi realizado o isolamento absoluto e para facilitar o procedimento restaurador obteve-se um guia de silicona a partir do segundo enceramento.

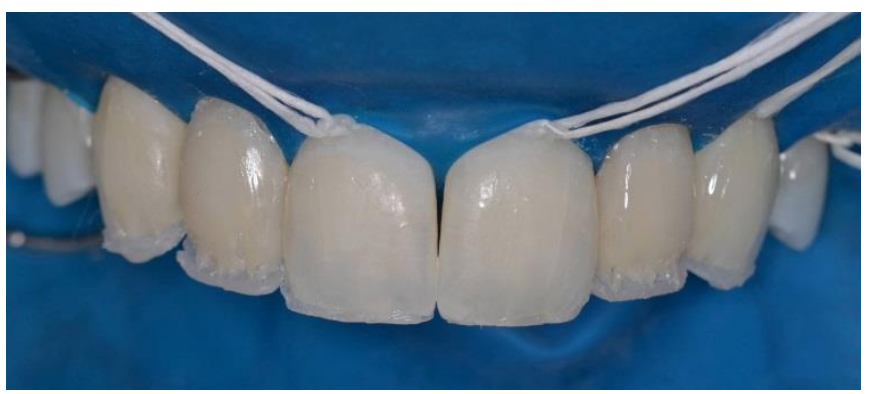

Figura 6- Porção de esmalte A2 (Empress) envolvendo o terço médio e cervical, sem recobrir os mamelos.

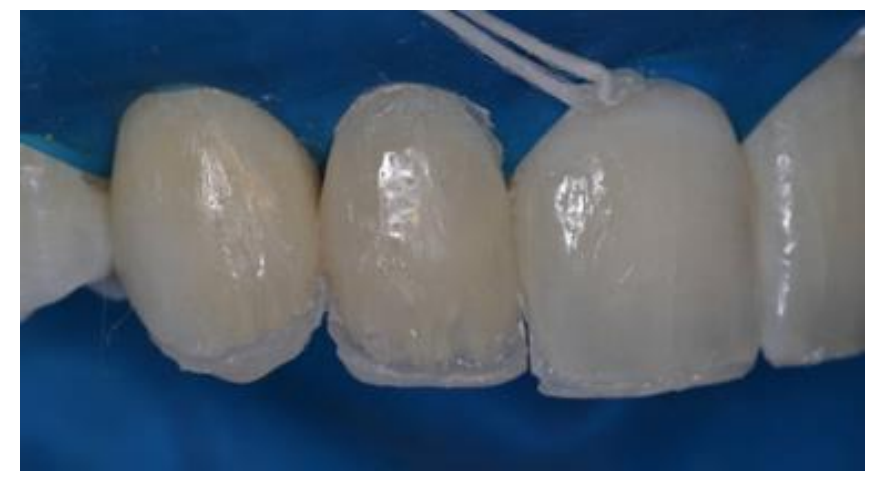

Figura 7- Resina de efeito IrB inserida ao redor e entre os mamelos dos dentes 13, 12, 22 e 23. 


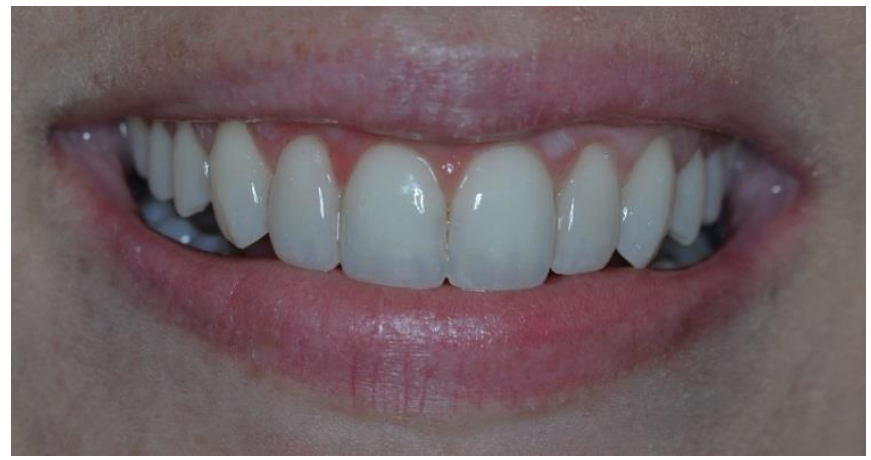

Figura 8- Aspecto final imediato.

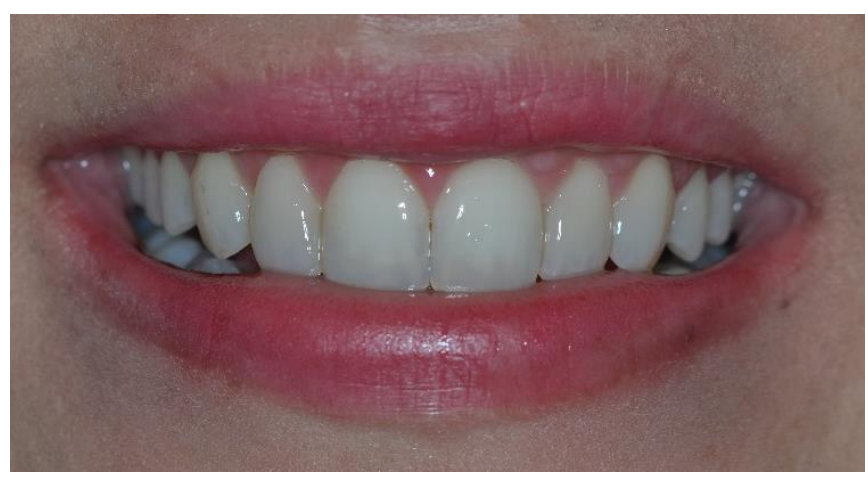

Figura 9- Controle de 7 meses.

\section{REFERÊNCIAS}

1. Ittipuriphat I, Leevailoj C. Anterior space management: interdisciplinary concepts. J Esthet Restor Dent. 2013; 25(1):16-30.

2. Rufenacht CR. Fundamentals of Esthetics. Carol Stream, IL: Quintessence Publish. 1990.

3. Regesi JA, Sciubba JJ. Patologia bucal: correlações clínico-patológicas. Rio de Janeiro: Guanabara Koogan; 1989.

4. Silva SBA, Argenta RMO, Machado R, Basso $\mathrm{R}$. Reconstrução de coroa com resina composta direta em dentes anteriores: relato de caso. Rev Faculd Odontol Passo Fundo. 2003;8(1):34-7.

5. Pedrini D, Jardim OS, Poi WR. Transformação de dente conóide e fechamento de diastema em clínica geral. Rev Faculd Odontol Lins. 2000;12(1):52-6.

6. Pena CE, Viotti RG, Dias WR, Santicci E, Rodrigues JA, Reis AF. Esthetic rehabilitation of anterior conoid teeth: comprehensive approach for improved and predictable results. Eur J Esthet Dent. 2009; 4(3):210-24.

7. Alvesalo L, Portin P. The inheritance pattern of missingpeg-shaped, and strongly mesio-distally reduced upperlateral incisors. Dental Aktieseiskabel. 1969;18(6): 563-75.
8. Staehle HJ, Wolff D, Frese C. More conservative dentistry: clinical long term results of direct composite resin restorations. Quintessence Inter. 2015 may; 46(5): 373-80.

9. Kurbad A. Planning and predictability of clinical outcomes in esthetic rehabilitation. Int J Comput Dent. 2015; 18(1):65-84.

10. Furuse AY, Franco EJ, Mondelli J. Esthetic and functional restoration for an anterior open occlusal relationship with multiple diastemata: a multidisciplinary approach. J Prosthet Dent. 2008;99(2):91-4.

11. Malik K, Tabiat-Pour S. The use of a diagnostic wax setup in aesthetic cases involving crown lengthening-a case report. Dent Update. 2010 jun; 37(5):303-4, 306-7.

12. Caudill R, Chiche G. Esthetique et gencive. In: Chiche GJ, Pinault A, Liger F. Esthetiqué et restaurations des dents anterieures. Paris: CdP; 1995.

13. Ozkan P, Kansu G, Ozak ST, Kurtulmus-Yilmaz S, Kansu P. Effect of bleaching agents and whitening dentifrices on the surface roughness of human teeth enamel. Acta Odontol Scand. 2013; 71(3-4):488-97.

14. Cvikl B, Lussi A, Moritz A, Flury S. Enamel surface changes after exposure to bleaching gels containing carbamide peroxide or hydrogen peroxide. Oper Dent. 2016 jan-feb;41(1):39-47.

15. Reis A, Higashi C, Loguercio AD. Re-anatomization of anterior eroded teeth by stratification with direct composite resin. J Esthet Restor Dent. 2009;21(5):30416.

16. Modena KCS, Colombini-Ishikiriama BL, Ishikiriama SK, Scotti CK, Nahsan FPS. Multidisciplinary esthetic approach for multiple anterior diastema: 36-month clinical performance. Braz Dent Sci. 2015 oct.dec;18(4):110-9.

17. Furuse AY, Herkrath FJ, Franco EJ, Benetti AR, Mondelli J. Multidisciplinary management of anterior diastemata: clinical procedures. Pract Proced Aesthet Dent. 2007; 19(3):185-91.

18. Pedroso LB, Barreto LF, Miotti LL, Nicoloso GF, Durand LB. Effect of a surface sealant on the color stability of composite resins after immersion in staining solution. Gen Dent. 2016 mar-apr;64(2):22-5.

19. Pallensen U, Van Djiken JW. A randomized controlled 30 year follow up of three conventional resin composites in Class II restorations. Dent Mater. 2015 Oct; 31(10):1232-44. 